\title{
Turbomachinery Blade Optimization Using the Navier-Stokes Equations
}

\author{
K.K. Chand \\ K.D. Lee \\ This paper was prepared for submittal to the \\ American Institute of Aeronautics and Astronautics \\ 36th Aerospace Sciences Meeting and Exhibit \\ Reno, $N V$ \\ January 12-15, 1998
}

December 1997

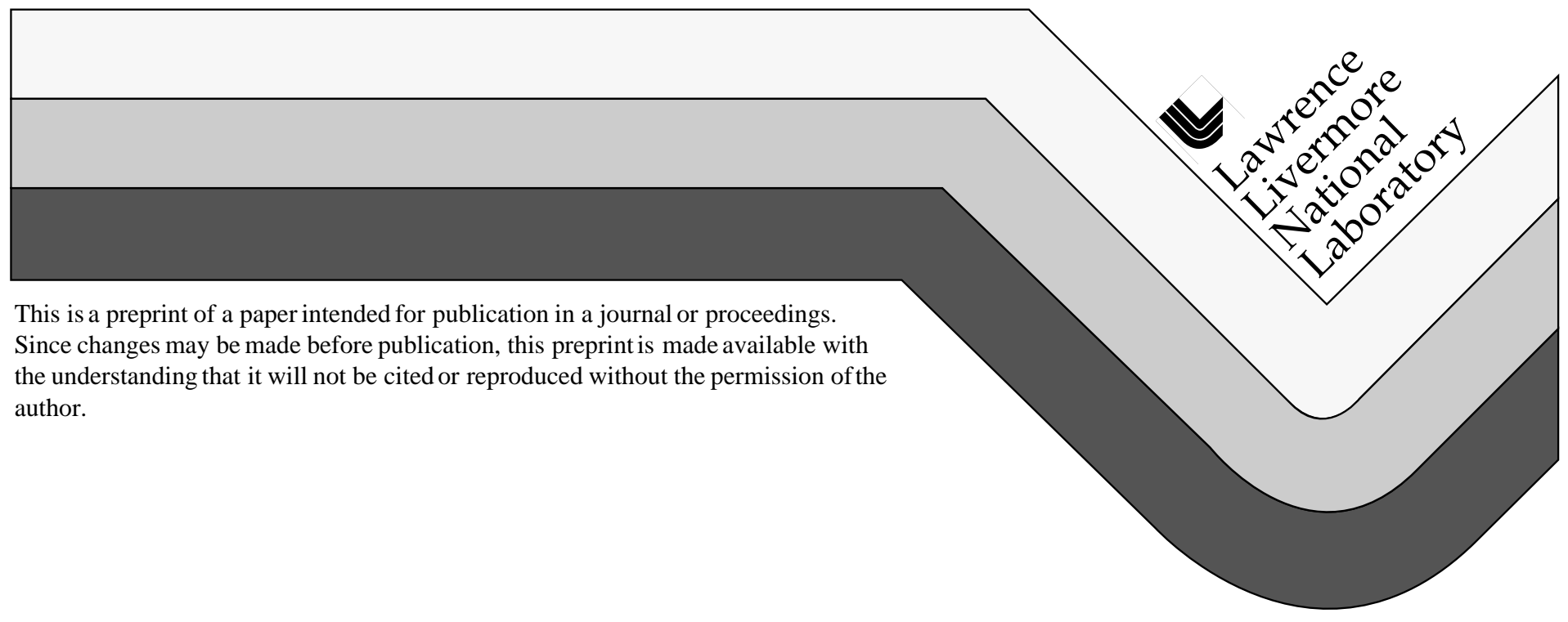




\section{DISCLAIMER}

This document was prepared as an account of work sponsored by an agency of the United States Government. Neither the United States Government nor the University of California nor any of their employees, makes any warranty, express or implied, or assumes any legal liability or responsibility for the accuracy, completeness, or usefulness of any information, apparatus, product, or process

disclosed, or represents that its use would not infringe privately owned rights. Reference herein to any specific commercial product, process, or service by trade name, trademark, manufacturer, or otherwise, does not necessarily constitute or imply its endorsement, recommendation, or favoring by the United States Government or the University of California. The views and opinions of authors expressed herein do not necessarily state or reflect those of the United States Government or the University of California, and shall not be used for advertising or product endorsement purposes. 


\title{
Turbomachinery Blade Optimization Using the Navier-Stokes Equations
}

\author{
K. K. Chand* and K. D. Lee** \\ Aeronautical and Astronautical Engineering \\ University of Illinois \\ Urbana, Illinois 61801
}

\begin{abstract}
A method is presented to perform aerodynamic design optimization of turbomachinery blades. The method couples a Navier-Stokes flow solver with a grid generator and numerical optimization algorithm to seek improved designs for transonic turbine blades. A fast and efficient multigrid, finite-volume flow solver provides accurate performance evaluations of potential designs. Design variables consist of smooth perturbations to the blade surface. A unique elliptic-hyperbolic grid generation method is used to regenerate a Navier-Stokes grid after perturbations have been added to the geometry. Designs are sought which improve a design objective while remaining within specified constraints. The method is demonstrated with two transonic turbine blades with different types and numbers of design variables.
\end{abstract}

\section{Introduction}

Development of future commercial transport aircraft requires new and higher performance jet engines. One approach to the development of such engines takes advantage of computational fluid dynamics (CFD). Traditionally, CFD has been used in an analysis mode for cut-and-try approaches to design. Specified geometries are analyzed for their performance and then modified and resubmitted for further analysis. While this methodology may be effective if the designs fall within a known experimental database, it becomes cumbersome and difficult when the design progresses outside the domain of the known database. Additionally, when the design space becomes complex, the cut-and-try approach can sacrifice the design effectiveness in time and cost. Reference 1 provides an overview of the methodology and difficulties of turbomachinery design. Clearly more automated design optimization technologies are necessary for reducing the time, and hence cost, necessary for the development of new turbomachinery components. Computational methods have matured to the point where CFD based numerical optimization becomes a viable approach to a more automated improvement of advanced turbomachinery components.

There are typically two approaches to using CFD for automated design: inverse design and constrained optimization. Inverse design is

* Research Assistant, Member AIAA

** Professor, Associate Fellow AIAA capable of finding the geometry that yields a pressure or velocity distribution specified by a designer. Several attempts have been made to perform inverse design of turbomachinery blades. Many of these attempts have involved the use of potential flow analysis of the flowfield in order to simplify the formulation and analysis of the $\operatorname{design}^{2,3}$. However, formulation of the inverse design problem is extremely difficult, especially when complicated flow physics, such as shock waves and shear layers, are involved. Unfortunately, transonic turbomachinery blade flows are dominated by such complicated physical phenomenon requiring the use of Euler and NavierStokes simulations. Quite often the necessary velocity or pressure distribution cannot be specified a priori. Additionally, there is no guarantee that a geometry exists for a specified distribution. Also, turbomachinery blade performance is often judged by parameters such as kinetic energy and total pressure loss coefficients which are not directly associated with the blade velocity or pressure distributions. Fortunately, another approach is available which circumvents the difficulties of inverse design.

Numerical optimization provides a rational and directed search through the design space. Instead of specifying the ultimate goal, as in inverse design, a search methodology is provided. CFD based optimization is capable of modifying a geometry in order to improve a design while remaining within specified design constraints ${ }^{4,5}$. In this manner, a specific design parameter may be 
improved without the degradation of other aspects of the design. For example, the objective may be to reduce a turbine's loss coefficient while constraining the blade loading to a specific value. Importantly, the resulting blade pressure or velocity distributions are not necessary a priori, but are determined as a result of the design process. Unfortunately, numerical optimization is often computationally expensive, particularly when the design involves nonlinear problems with many design variables and constraints. However, the efficiency of modern flow solvers, as well as the computational power available today, alleviates this drawback to optimization.

In order to provide a reliable and accurate design the numerical optimization must be presented with an accurate representation of the flow field. Transonic turbomachinery flows, however, contain a variety of complicated phenomenon including shock waves, turbulent boundary layers and wakes. Clearly the full potential equations cannot accurately represent these flows. Comparatively few attempts have been made to utilize Euler or Navier-Stokes flow analysis for the numerical optimization of turbomachinery blades. A primary reason for this involves both the maturity and cost of NavierStokes analysis. Reference 6 used Euler analysis to search for shockless compressor blade designs. Reference 7 utilized an Euler flow solver to reduce the loss coefficient of turbine blades. The present study follows the design approach of Reference 7 while using Navier-Stokes analysis to more accurately predict the turbine blade performance.

The present study develops and presents a turbomachinery design tool based on constrained optimization with Navier-Stokes flow analysis. A novel elliptic-hyperbolic grid generator is incorporated into the design to ensure that grid quality is maintained as new geometries are investigated. The method is tested and evaluated by implementing it on three turbine blades. The present paper presents design results for two turbine blades.

\section{Tools and Methods}

\section{Flow Analysis}

The optimization utilizes a 2D, multigrid Navier-Stokes flow code that solves the Reynoldsaveraged Navier-Stokes equations in conservative form using a cell centered finite-volume scheme $^{8,9}$. Runge-Kutta integration is used with local timestepping to produce a steady state solution. Both second- and fourth-order numerical dissipations are used to ensure stability. To improve convergence and efficiency, implicit residual smoothing and a multi-level multigrid method are implemented. Turbulence closure is provided by the Baldwin-Lomax eddy viscosity model ${ }^{10}$. Boundary conditions along the blade are provided by zero velocity at the walls and zero pressure gradient through the boundary layer. Inlet and outlet boundary conditions are determined by a one dimensional analysis of the Riemann variables.

\section{Elliptic-Hyperbolic Grid Generation}

Grid quality is critically important to maintaining the accuracy and efficiency of a Navier-Stokes flow solution. The flowfield around a turbine blade presents several difficulties in grid generation. Quality grids with good grid density and orthogonality are hard to generate due to the high camber, rounded leading and trailing edges, and narrow pitch in the turbine geometry. Hyperbolic grid generation methods produce quality grids in the near-field but may not properly handle the periodic boundaries. Meanwhile, elliptic methods are excellent in gridding the confined in-field but have difficulty controlling grid quality in the near-field. This grid problem is circumvented by utilizing a unique grid generation method that combines elliptic and hyperbolic grid generation.

The elliptic-hyperbolic hybrid (EHH) grid generation method of Bacon and Lee ${ }^{11}$ has the ability to vary the amount of elliptic and hyperbolic characteristics of the grid. This method is formulated by linearly combining the elliptic and hyperbolic operators as

$$
(1-\mu) \mathrm{H}(\mathbf{R})+\mu \mathrm{E}(\mathbf{R})=0
$$

where

$$
\begin{aligned}
& \mu=\text { weighting parameter } \\
& \mathrm{H}(\mathbf{R})=\text { hyperbolic function } \\
& \mathrm{E}(\mathbf{R})=\text { elliptic function } \\
& \mathbf{R}=[\mathrm{x}, \mathrm{y}]^{\mathrm{T}}
\end{aligned}
$$

Importantly, $\mu$ can be varied throughout the domain so that the grid is predominantly hyperbolic near the walls and elliptic in the middle of the domain. Not only does the EHH method produce more orthogonal grids in the difficult trailing edge regions, it preserves the smooth nature of elliptic grids throughout the domain. In addition, EHH grid generation is robust and computationally efficient making it ideal for 
repeated application during a numerical optimization.

Minimize: $\quad F(\mathbf{X})$

\section{Numerical Optimization}

Subject to:

$$
\begin{array}{ll}
\mathrm{g}_{\mathrm{j}}(\mathbf{X}) \leq 0 & \mathrm{j}=1, \mathrm{~J} \\
\mathrm{X}_{\mathrm{k}}^{\mathrm{L}} \leq \mathrm{X}_{\mathrm{k}} \leq \mathrm{X}_{\mathrm{k}}^{\mathrm{U}} & \mathrm{k}=1, \mathrm{~K}
\end{array}
$$

The present method utilizes a commercial optimization package to provide a robust and efficient algorithm for the numerical optimization $^{12}$. A general presentation of a constrained optimization problem is:

$\mathbf{X}$ represents the vector of design variables $X_{k}$. $F$ is the objective function, and the $g_{j}$ are the constraint functions. $\mathrm{J}$ represents the number of constraints and $\mathrm{K}$ the number of design variables. Superscripts $\mathrm{L}$ and $\mathrm{U}$ denote the lower and upper side constraints on the design variables. Typically, equality constraints are imposed by using two inequality constraints.

Using this information, the first step in nonlinear optimization is to form the Langrangian:

$$
L(\mathbf{X}, \lambda)=F(\mathbf{X})+\sum_{\mathrm{j}=1}^{\mathrm{J}} \lambda_{\mathrm{j}} \mathrm{g}_{\mathrm{j}}(\mathbf{X})
$$

where the $\lambda_{j}$ are the Lagrange multipliers. A local optimum has been reached when the gradient of the Lagrangian equals zero, which is known as the Kuhn-Tucker condition:

$$
\nabla F(\mathbf{X})+\sum_{\mathrm{j}=1}^{\mathrm{J}} \lambda_{\mathrm{j}} \nabla \mathrm{g}_{\mathrm{j}}(\mathbf{X})=0
$$

$\nabla F$ and $\nabla g_{\mathrm{j}}$ are the sensitivities of the objective function and constraints, respectively, to changes in the design variables. In the current work, sensitivities are calculated using a finite difference technique. For example, the sensitivity of $F$ for the kth design variable is:

$$
\frac{\partial F}{\partial \mathrm{X}_{\mathrm{k}}}=\frac{F\left(\mathbf{X}+\Delta \mathrm{X}_{\mathrm{k}}\right)-F(\mathbf{X})}{\Delta \mathrm{X}_{\mathrm{k}}}
$$

Optimization begins with an initial choice for the design variables. For example, the design process starts from a known turbine blade. The optimization then proceeds to update the design variables by finding a search direction and moving the design variables along this direction The distance traveled along this direction is the step size.

$$
\mathbf{X}^{\mathrm{n}+1}=\mathbf{X}^{\mathrm{n}}+\beta \mathbf{S}^{\mathrm{n}}
$$

where $\mathbf{S}$ is the search direction, $\beta$ the step size and $\mathrm{n}$ the current design iteration. Eq. (6) is applied 
iteratively until the Kuhn-Tucker condition of Eq. 4 is satisfied.

A search direction must be both usable and feasible. Usable search directions are those which reduce the objective function and feasible directions satisfy the constraints. Mathematically,

$$
\begin{aligned}
& \nabla F(\mathbf{X}) \bullet \mathbf{S} \leq 0 \\
& \nabla \mathrm{g}_{\mathrm{j}}(\mathbf{X}) \bullet \mathbf{S} \leq 0
\end{aligned}
$$

A constraint is considered active when it violates the feasibility condition within a specified tolerance. The Fetcher-Reeves conjugate gradient method is used to find the search direction when no constraints are active. This method is a modified form of the steepest descent algorithm which biases the current search direction with the previous search direction. In other words, the determination of the new search direction utilizes information from the previous direction.

When one or more constraints are active the determination of the search direction is more difficult due to the restrictions imposed by the constraints. In this case, minimizing Eq. (7) subject to the constraints of Eq. (8) yields the search direction. This procedure is known as a suboptimization and is also performed by the commercial optimization package.

It is important to realize that the optimality condition of Eq. (4) signifies only a local optimum. In practice it is very difficult to obtain a global optimum. A better initial condition may produce a better design. In general, the present design goal is to improve the performance of an existing configuration although there is no guarantee "global optimality."

\section{Design Variables}

Since turbomachinery flows contain many complicated and interacting phenomenon, careful choice of the design variables and constraints can significantly affect the optimization results. The cost of optimization increases by increasing the number of design variables and constraints. Alternatively, if the design variables and constraints are carefully chosen the design procedure becomes more effective and efficient.

In the current study, design variables consist of smooth perturbations to the initial turbine blade. These perturbations are added to the upper and lower blade surfaces in a direction normal to the camber line. The total perturbation, $\Delta n$, is defined as

$$
\Delta n=\sum_{\mathrm{k}=1}^{\mathrm{K}} \mathrm{X}_{\mathrm{k}} \mathrm{f}_{\mathrm{k}}(\mathrm{s})
$$

where the $f_{k}(s)$ are smooth functions representing the shape of the perturbations and $\mathrm{s}$ is the location along the camber line. Here, the design variables, $\mathrm{X}_{\mathrm{k}}$, are weightings for each shape function.

\section{Demonstration}

Demonstrations are conducted using two initial geometries: the VKI-rotor turbine, and the VKILS82 turbine blade ${ }^{13}$. Initial grids for these blades are illustrated in Figure 1. The design goal is to reduce the loss coefficient, defined in Eq. (10), without decreasing the blade area, A, mass flow, M, and loading, L. These constraints are presented in Eq. (11). In these equations, $v_{2}$ is the exit velocity and subscript 0 represents the initial design value. Preventing a decrease in the area helps to avoid thin areas in the blade which cause structural problems at the high temperatures and loadings commonly found in turbines. The latter two constraints preserve the design requirements specified for the turbine.

$$
\begin{gathered}
C_{\text {loss }}=1-\frac{v_{2}^{2}}{v_{2 i s}^{2}} \\
1-\frac{A}{A_{0}} \leq 0,1-\frac{M}{M_{0}} \leq 0,1-\frac{L}{L_{0}} \leq 0
\end{gathered}
$$

During the optimization, the grid is regenerated after adding each perturbation to the geometry. In order to maintain computational efficiency, flow solutions are restarted from the best available solutions. Solutions are considered converged when the maximum residual is reduced by four orders of magnitude from the free-stream residual after a specified number of iterations have been completed.

\section{$\underline{\text { VKI-rotor }}$}

The shape functions defining the geometry perturbations for this turbine blade are the patched polynomials given below:

$$
\begin{array}{r}
f_{k}=1-\left[\frac{\left(s_{k}-s\right)}{s_{k}}\right]^{2}\left[1+\frac{A s}{s_{k}\left(1-s_{k}\right)^{2}}\right] \quad(14 \mathrm{a}) \\
\text { for } 0 \leq s \leq s_{k}
\end{array}
$$




$$
f_{k}=1-\left[\frac{\left(s-s_{k}\right)}{\left(1-s_{k}\right)}\right]^{2}\left[1+\frac{B s(1-s)}{s_{k}^{2}\left(1-s_{k}\right)}\right]
$$

$$
\text { for } s_{k} \leq s \leq 1
$$

where

$$
\begin{aligned}
& \mathrm{A}=\max \left(0,1-2 \mathrm{~s}_{\mathrm{k}}\right) \\
& \mathrm{B}=\max \left(0,2 \mathrm{~s}_{\mathrm{k}}-1\right)
\end{aligned}
$$

In the above equations, $s_{k}$ represents the location of the maximum of the functions.

The first design utilizes six design variables, three for the upper surface and three for the lower. Values for $\mathrm{s}_{\mathrm{k}}$ used for this case are : 0.25, 0.5, 0.75 for both the upper and lower surfaces. This turbine operates at an exit isentropic Mach number of 1.02 and an inlet flow angle, $\alpha$, of $30^{\circ}$. The results of the optimization are summarized in Table 1 and Figure 2. A $22.4 \%$ reduction of the loss coefficient was produced by the design. However, some of the constraints were slightly violated. Figure $2 \mathrm{c}$ shows the slight change in the initial geometry as a result of the design. By examining the isentropic Mach number distributions and the pressure contours it seems that the new design reduced the strength of the shock waves in order to decrease the loss coefficient.

In an effort to improve the design, ten design variables are used, five for each surface. Their $s_{k}$ values, for both surfaces, are: $0.15,0.25,0.5,0.75$, 0.85 . This time only a $16.1 \%$ decrease in the loss coefficient is seen. On the other hand, the constraints were violated less during the design. These results are seen by examining Table 2 and Figure 3. The geometry changes are depicted in Figure $3 \mathrm{c}$ while the effects of the design changes on the isentropic Mach number distributions and flowfield are illustrated in Figures $3 \mathrm{~d}$, e and f. Again, the strength, and hence detriment, of the shock waves were reduced as a result of the design.

\section{VKI-LS82 turbine}

For the VKI-LS82 turbine, a different set of design variables is demonstrated. The Hicks-Henne sinusoidal functions, given in Eq. 15, are used ${ }^{14}$.

$$
\begin{aligned}
& f_{1}(s)=s^{25}(1-s) \exp (-20 s) \\
& f_{\mathrm{k}}(s)=\sin ^{3}\left(\pi s^{e(k)}\right), \mathrm{k}>1
\end{aligned}
$$

where

$$
e(k)=\frac{\log (0.5)}{\log \left(s_{\mathrm{k}}\right)}
$$

Again, $s_{k}$ denotes the location of the maximum of the functions and $\mathrm{s}$ the location along the camber line. For this blade, the exit isentropic Mach number was 1.43 with an $\alpha$ of $0^{\circ}$. Two attempts were made using six and ten design variables with the same $s_{k}$ values used for the corresponding VKIrotor cases.

Tables 3 and 4 summarize the results using six and ten design variables respectively. Figures 4 and 5 depict the changes in the objective, constraints, geometry, isentropic Mach number distributions and flowfield for these cases. Again, the optimized designs generate weaker shocks, and hence less losses due to shocks.

In this case, the use of more design variables produces a much better design than using only six. With six variables, only a $6.3 \%$ improvement in the objective function is achieved. With ten, the design is $9.3 \%$ better than the original geometry. Unfortunately, a high cost is involved with using more design variables. While it only took $28 \mathrm{CPU}$ minutes on a Cray C-90 to perform the design with six design variables, it took 66 minutes using ten. More than twice as much computer time is required in order to gain an extra $3.0 \%$ improvement in the design.

\section{Concluding Remarks}

The presented method can consistently find improved designs for transonic turbine blades. The optimization algorithm provides a means of improving the design objective while maintaining crucial constraints. A Navier-Stokes solver assisted by a unique, high quality grid generator ensures that the optimization is efficient and accurate. Cost and efficiency of the method is shown to be strongly affected by the number of design variables and less influenced by the choice of shape functions. Future directions include a study of the effects of turbulence modeling and grid density on turbine design. Also, this work will be extended to the optimization of transonic compressor blades.

\section{References}

1. Jennions, I. K., "Elements of Modern Turbomachinery Design System," AGARD Lecture Series 195, AGARD-LS-195, May 1994.

2. Sanz, J. M., "Design of Supercritical Cascades With High Solidity," AIAA Journal, Vol. 21, No. 9, September 1983, pp. 1289-1293. 
3. Dulikravich, G. S. and Sobieczy, H., "Shockless Design and Analysis of Transonic Shapes," AIAA Journal, Vol. 20, No. 11, November 1982, pp. 1572-1587.

4. Shelton, M. L., Gregory, B. A., Lamson, S. H., Moses, H. L., Doughty, T. L. and Kiss, T., "Optimization of a Transonic Turbine Airfoil Using Artificial Intelligence, CFD, and Cascade Testing," ASME Paper 93-GT-161, International Gas Turbine and Aeroengine Congress and Exposition, May 1993.

5. Sanger, N. L., "The Use of Optimization Techniques to Design Controlled Diffusion Compressor Blading," Journal of Engineering for Power, Vol. 105, April 1983, pp. 256-264.

6. Tong, S. S. and Thompkins, W. T., "A Design Calculation Procedure for Shock-Free or Strong Passage Shock Turbomachinery Cascades," Journal of Engineering for Power, Vol. 105, April 1983, pp. 369-376.

7. Eyi, S. and Lee, K. D., "Transonic Turbomachinery Blade Design Using Optimization," AIAA Paper 97-0159, 35th Aerospace Sciences Meeting \& Exhibit, Reno, NV, January 1997.

8. Martinelli, L., Jameson, A., and Grasso, F., "A Multigrid Method for the Navier-Stokes Equations," AIAA Paper 86-0208, 1986.

9. Arnone, A. and Swanson, R. C., "A NavierStokes Solver for Cascade Flows," NASA-CR181682, 1988.

10. Baldwin, B. S., and Lomax, H., "Thin Layer Approximation and Algebraic Model for Separated Turbulent Flows," AIAA 78-0257, AIAA 16th Aerospace Sciences Meeting, Huntsville, AL, January 1978.

11. Bacon, J. A., Henderson, T. L., and Lee, K. D., "Grid Generation Using an EllipticHyperbolic Hybrid Method," Proceedings of 4th International Conference on Numerical Grid Generation in Computational Fluid Dynamics and Related Fields, Swansea, UK, April 1994, pp. 1-13.

12. Vanderplaats, G.N., Numerical Optimization Techniques for Engineering Design, McGraw-Hill Book Company, 1984.

13 "Workshop on 2D and 3D Flow Calculations in Turbine Bladings," VKI Report, LS-82-05, April 1982.

14. Hicks, R. M., and Henne, P. A., "Wing Design by Numerical Optimization," Journal of Aircraft, Vol. 15, 1978, pp. 407-412.

This work was performed under the auspices of the U.S. Dept. of Energy at LLNL under contract no. W-7405-Eng-48. 
Table 1. Design optimization of the VKI-rotor blade using six design variables

\begin{tabular}{|l|c|c|c|}
\hline & Initial & Design & Change (\%) \\
\hline C $_{\text {loss }}$ & 0.0853 & 0.0662 & -22.43 \\
\hline Area & 0.2787 & 0.2790 & 0.10 \\
\hline Mass flow & 0.2337 & 0.2347 & 0.44 \\
\hline Blade loading & 0.2460 & 0.2513 & 2.13 \\
\hline
\end{tabular}

Table 2. Design optimization of the VKI-rotor blade using ten design variables

\begin{tabular}{|l|c|c|c|}
\hline & Initial & Design & Change (\%) \\
\hline Closs $_{\text {Area }}$ & 0.0853 & 0.0716 & -16.05 \\
\hline Mass flow & 0.2787 & 0.2790 & 0.10 \\
\hline Blade loading & 0.2337 & 0.2342 & 0.21 \\
\hline
\end{tabular}

Table 3. Design optimization of the VKI-LS82 blade using six design variables

\begin{tabular}{|l|c|c|c|}
\hline & Initial & Design & Change (\%) \\
\hline$C_{\text {loss }}$ & 0.0902 & 0.0846 & -6.26 \\
\hline Area & 0.1579 & 0.1579 & 0.00 \\
\hline Mass flow & 0.0882 & 0.0892 & 1.16 \\
\hline Blade loading & 0.1139 & 0.1156 & 1.44 \\
\hline
\end{tabular}

Table 4. Design optimization of the VKI-LS82 blade using ten design variables

\begin{tabular}{|l|c|c|c|}
\hline & Initial & Design & Change (\%) \\
\hline Closs $_{\text {lore }}$ & 0.0902 & 0.0819 & -9.29 \\
\hline Area & 0.1579 & 0.1577 & -0.14 \\
\hline Mass flow & 0.0882 & 0.0907 & 2.78 \\
\hline Blade loading & 0.1139 & 0.1170 & 2.66 \\
\hline
\end{tabular}

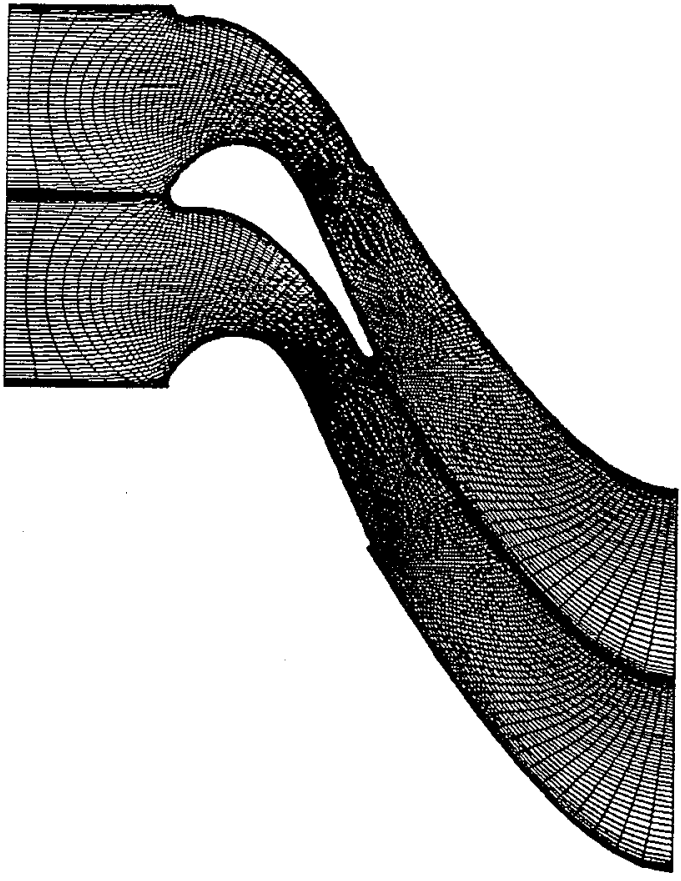

(a) VKI-rotor grid $(129 \times 65)$

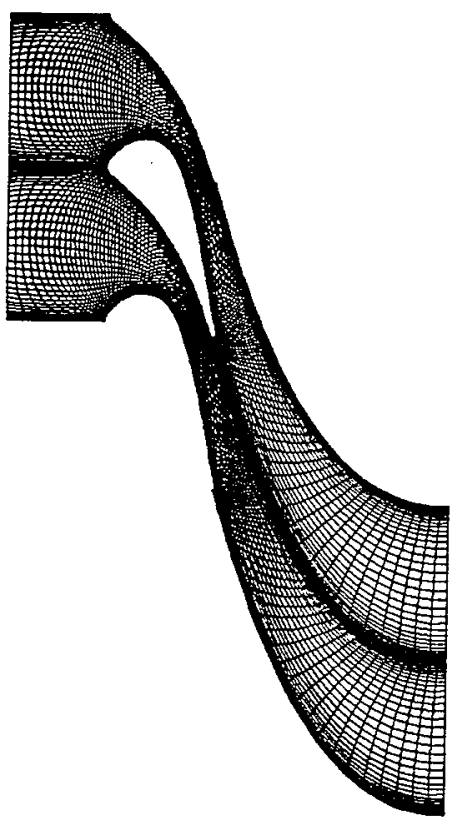

(b) VKI-LS82 grid $(129 \times 65)$

Figure 1. Grids for the initial geometries 


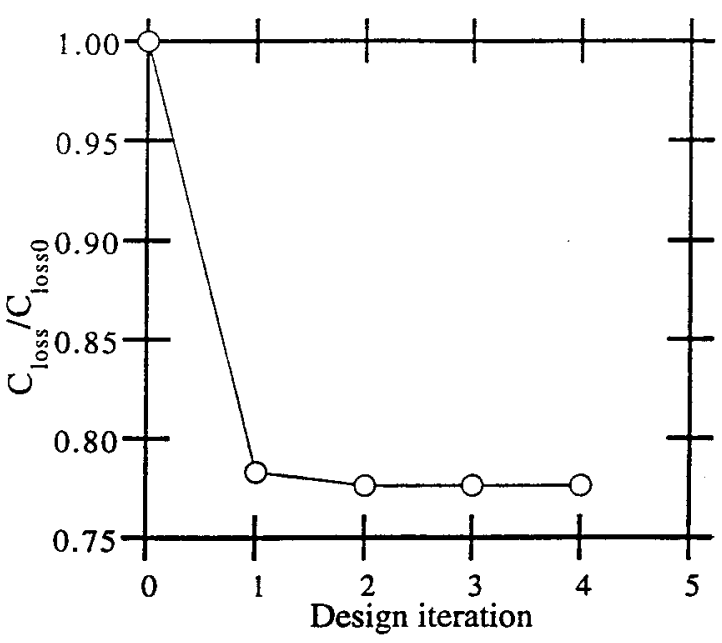

(a) Loss coefficient

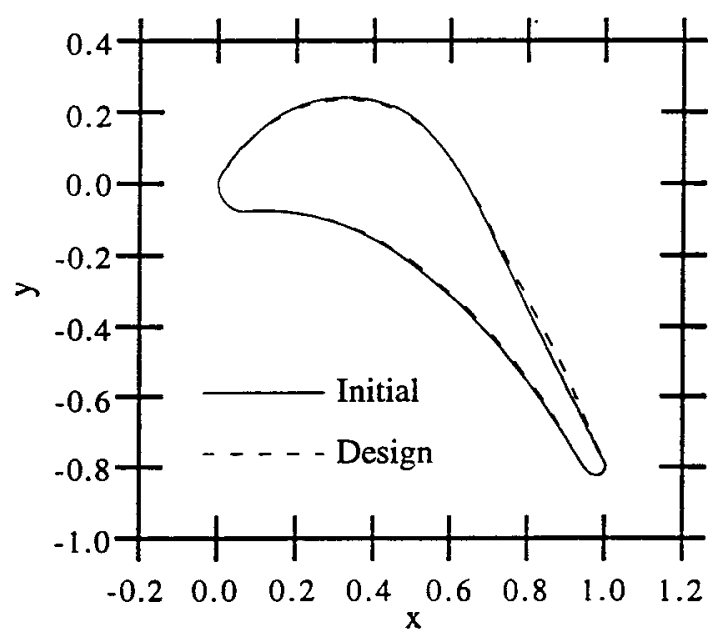

(c) Blade geometry

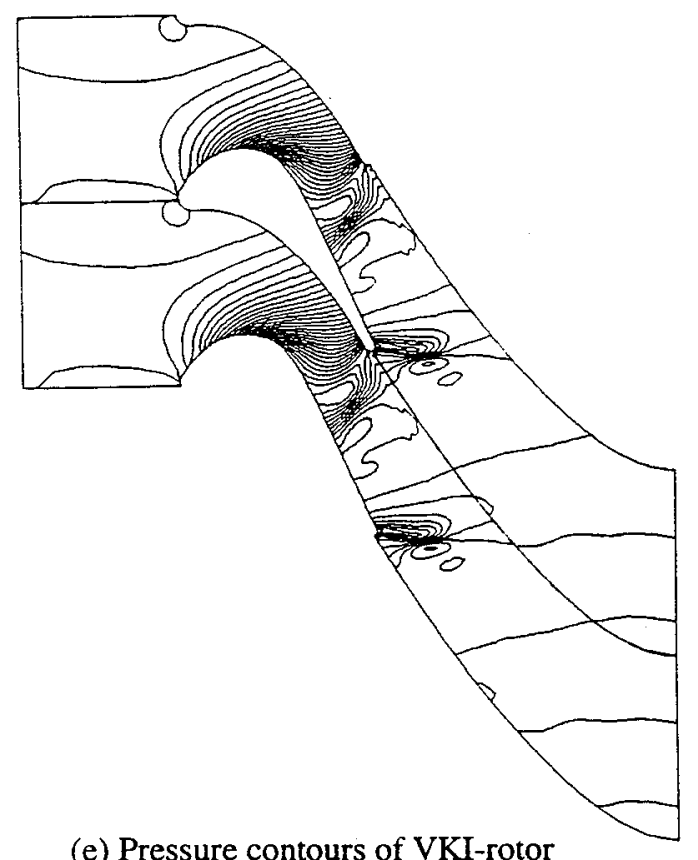

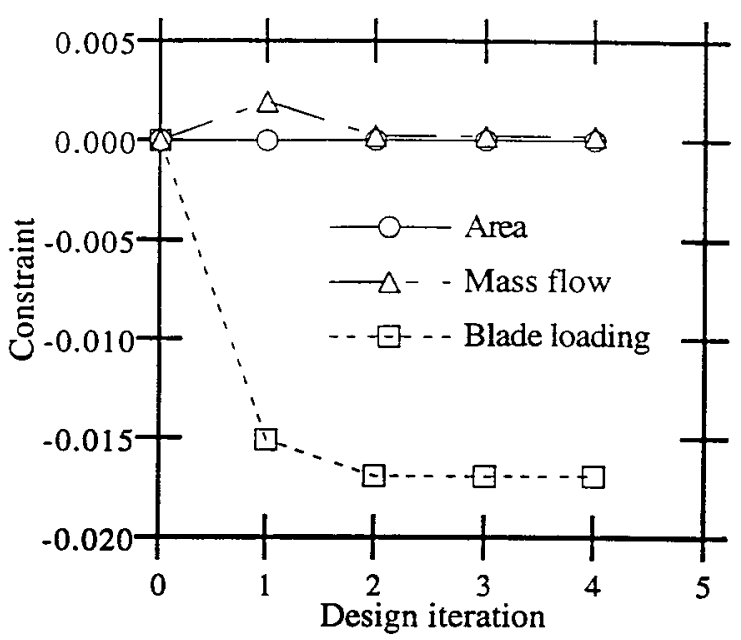

(b) Constraints

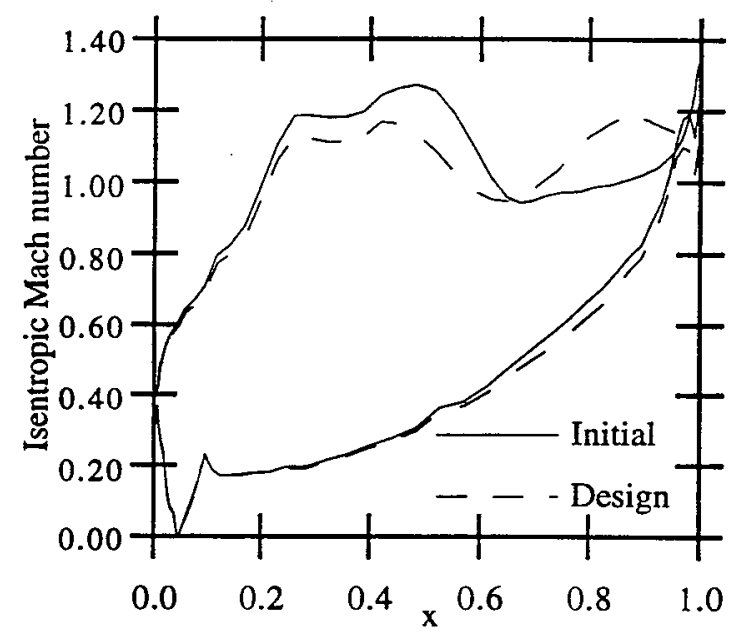

(d) Isentropic Mach number

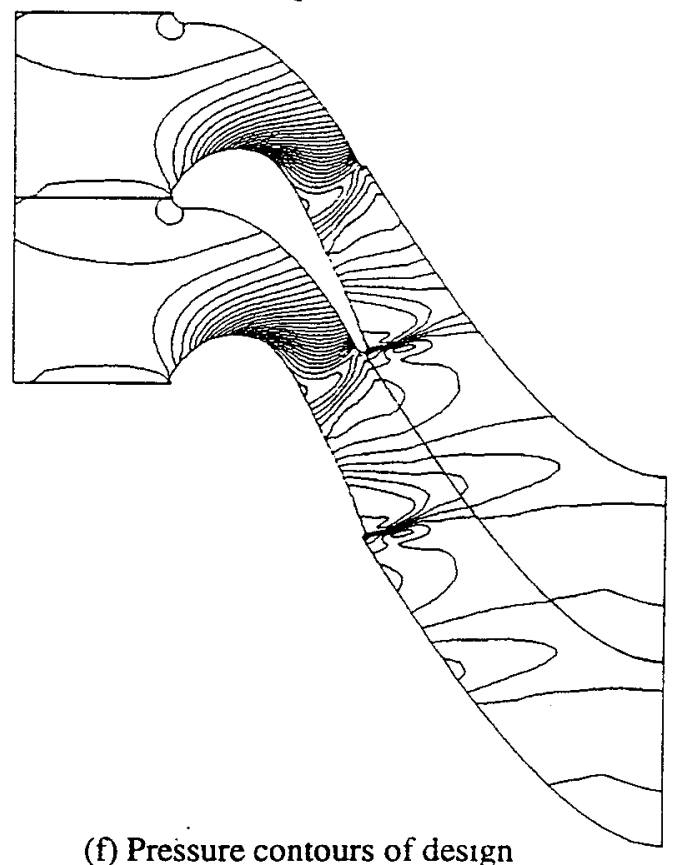

Figure 2. Design optimization of the VKI-rotor turbine blade using six design variables. 


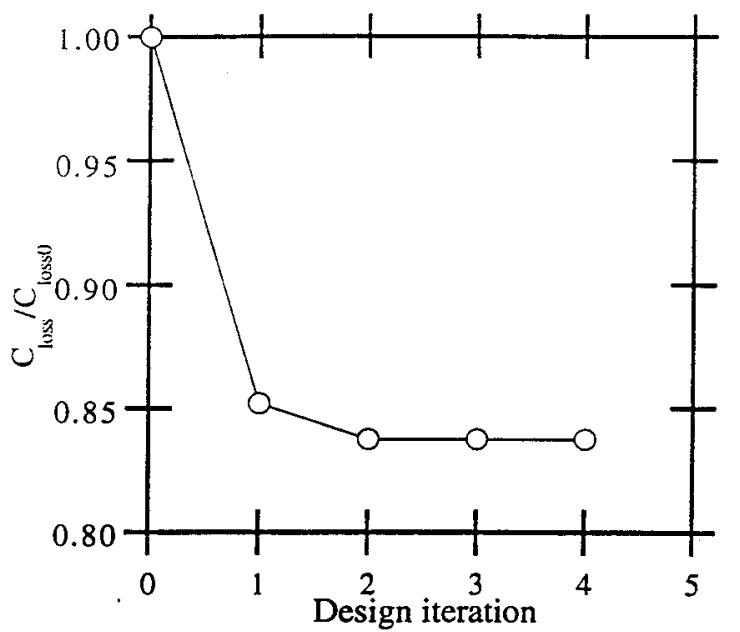

(a) Loss coefficient

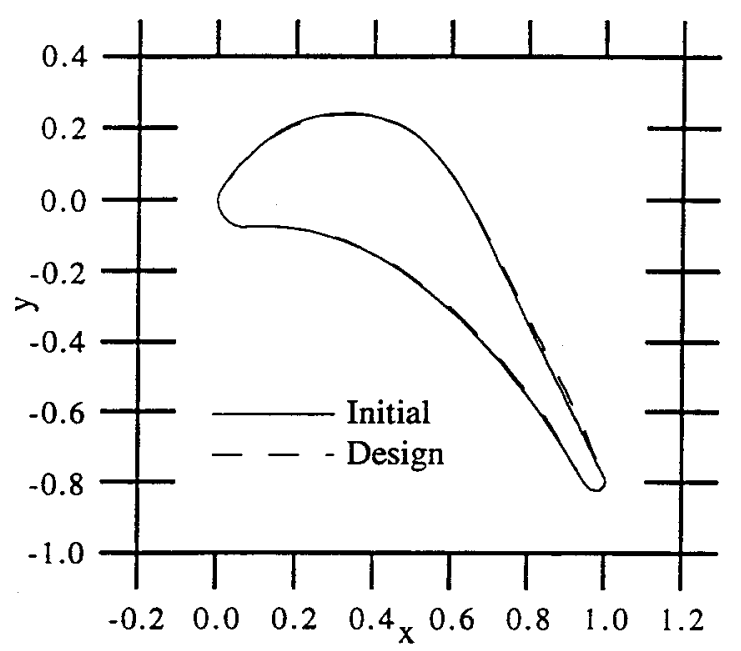

(c) Blade geometry

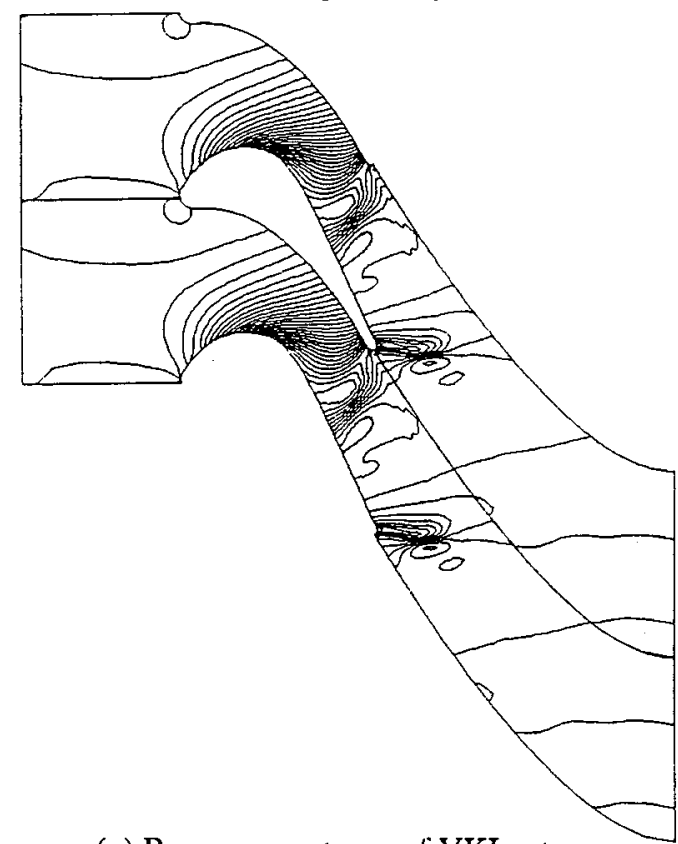

(e) Pressure contours of VKI-rotor

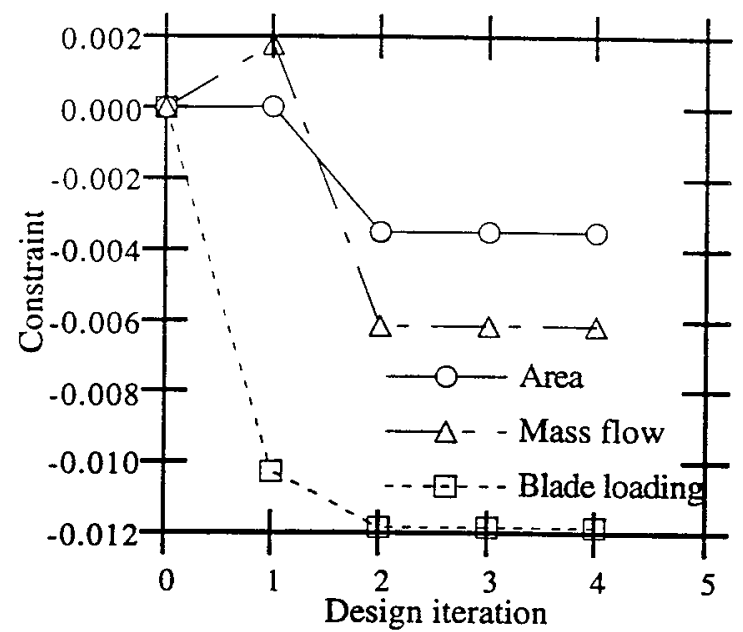

(b) Constraints

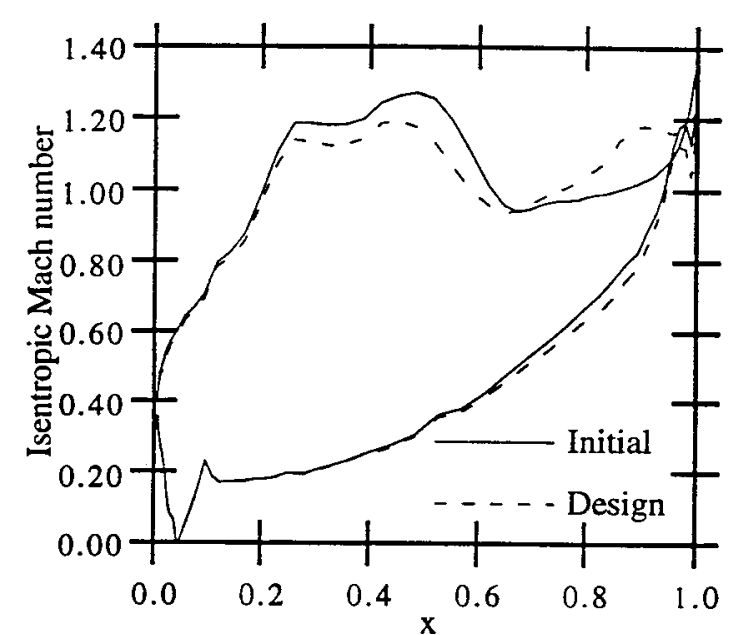

(d) Isentropic Mach number

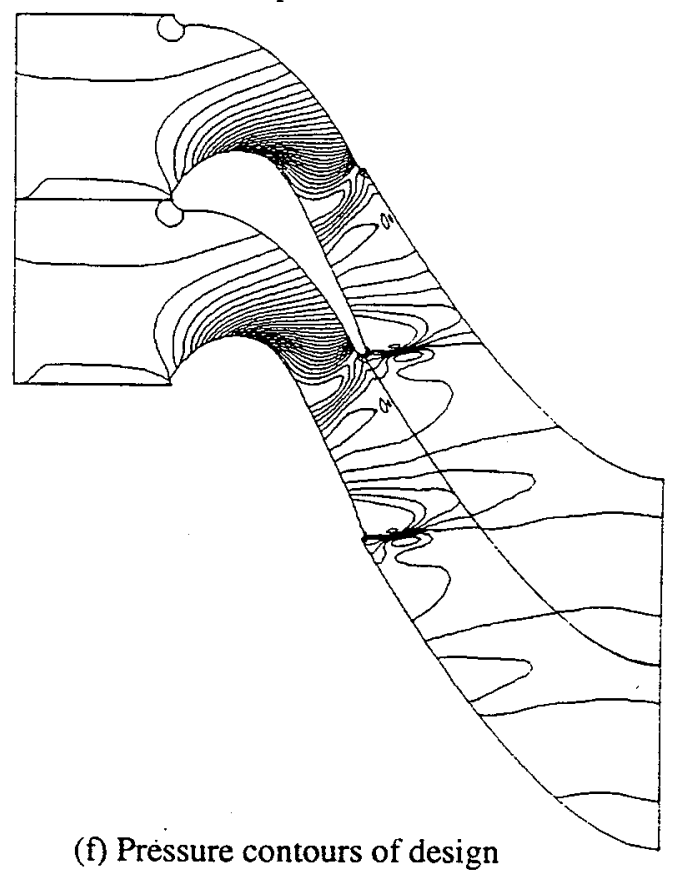

Figure 3. Design optimization of the VKI-rotor turbine blade using ten design variables. 


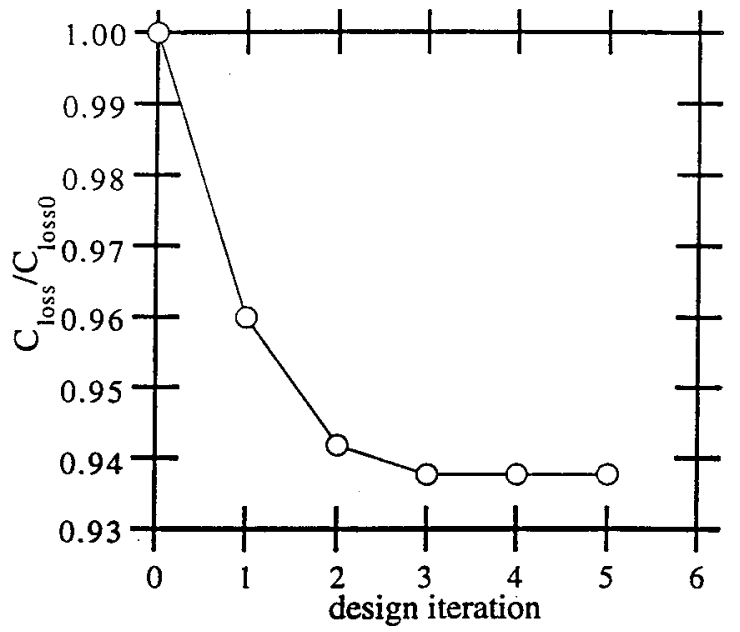

(a) Loss coefficient

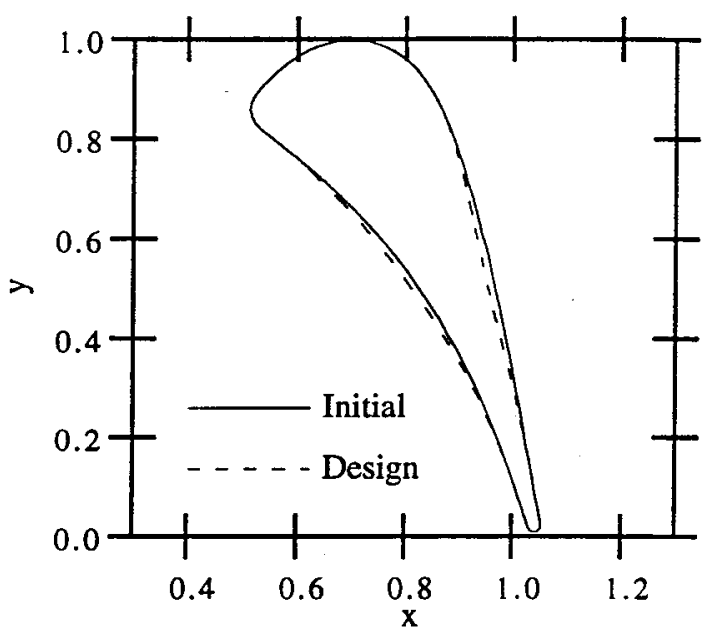

(c) Blade geometry

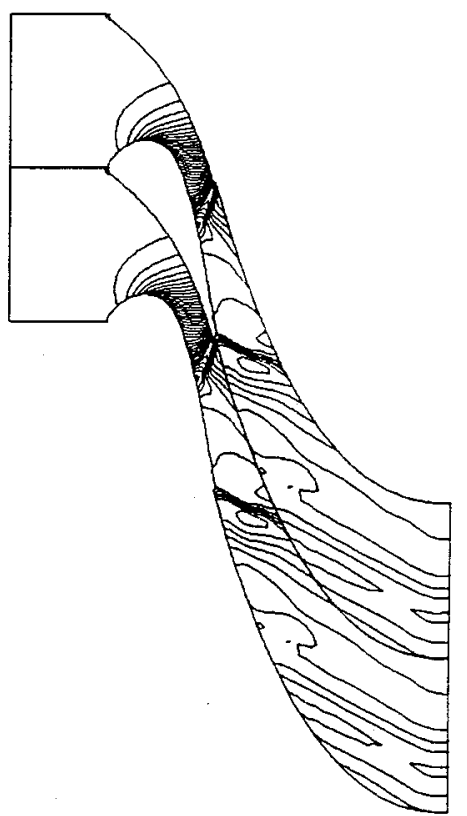

(e) Pressure contours of VKI-LS82 turbine

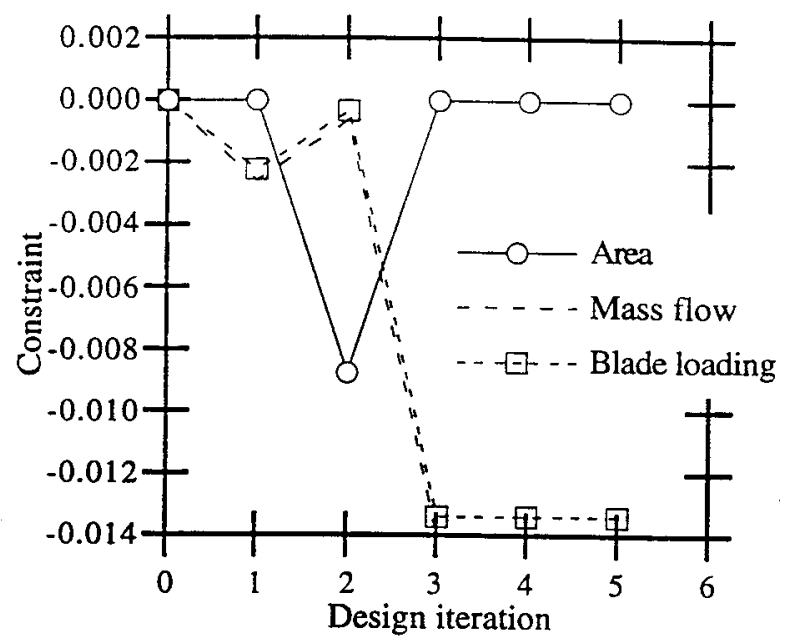

(b) Constraints

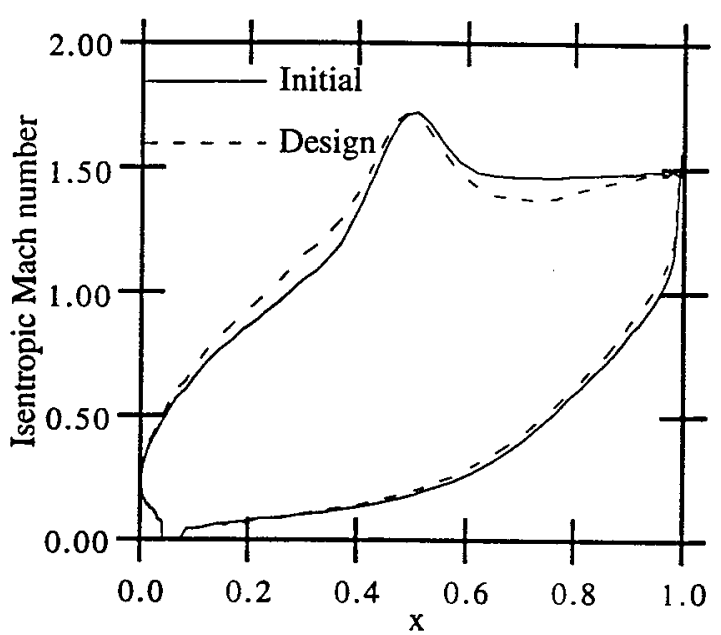

(d) Isentropic Mach number

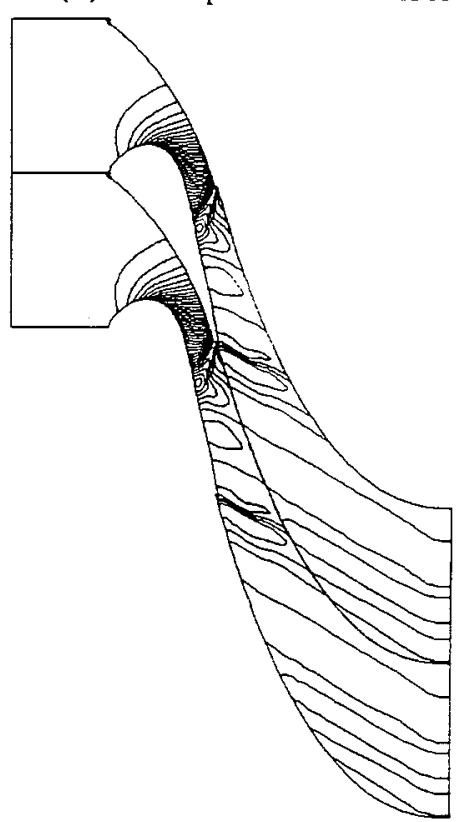

(f) Pressure contours of design

Figure 4. Design optimization of the VKI-LS82 turbine blade using six design variables. 


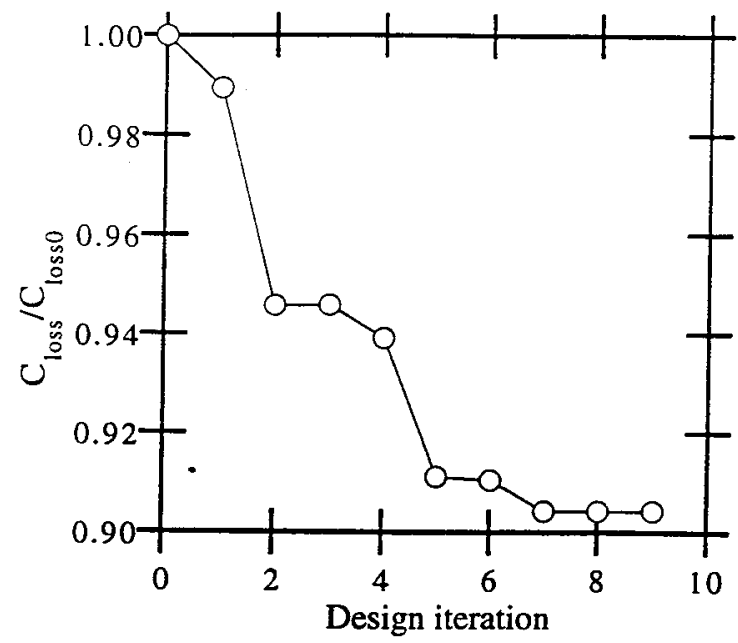

(a) Loss coefficient

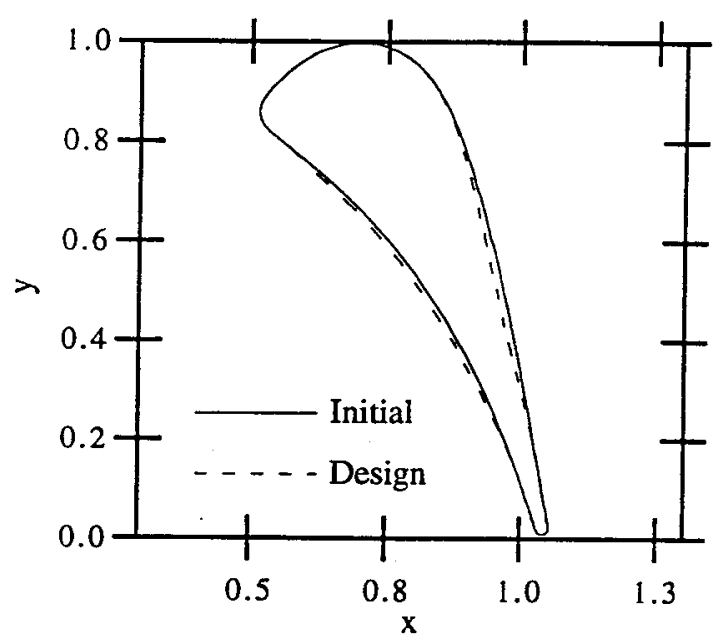

(c) Blade geometry

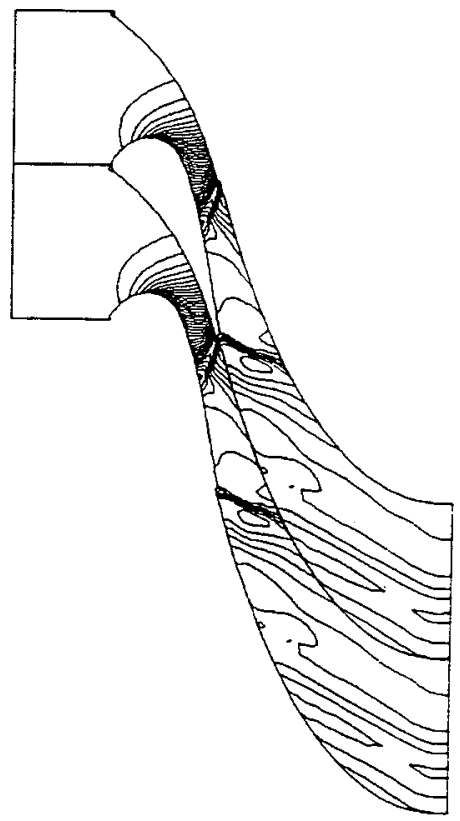

(e) Pressure contours of VKI-LS82 turbine

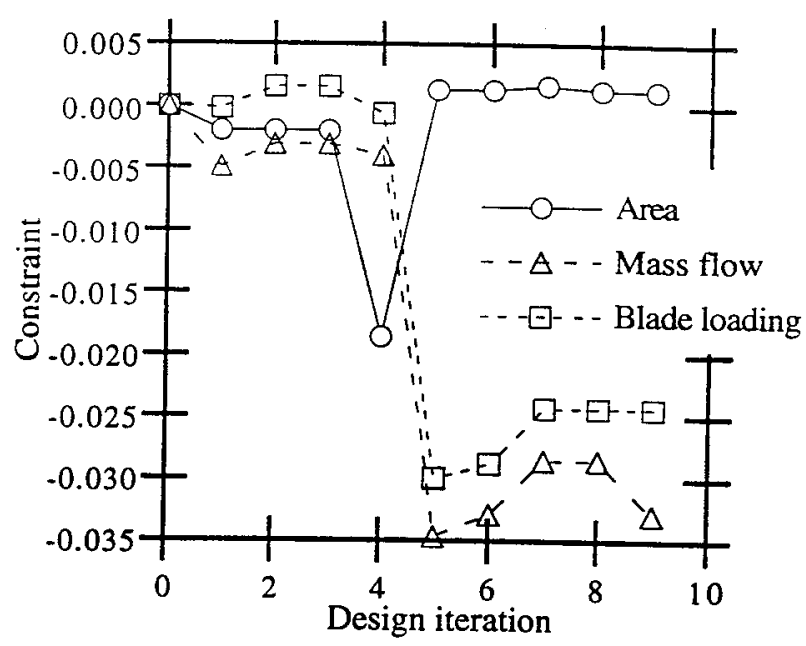

(b) Constraints

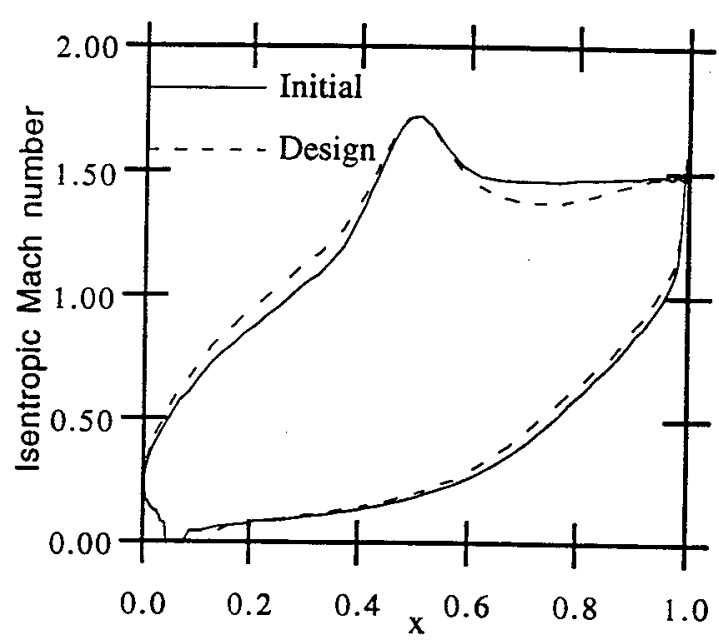

(d) Isentropic Mach number

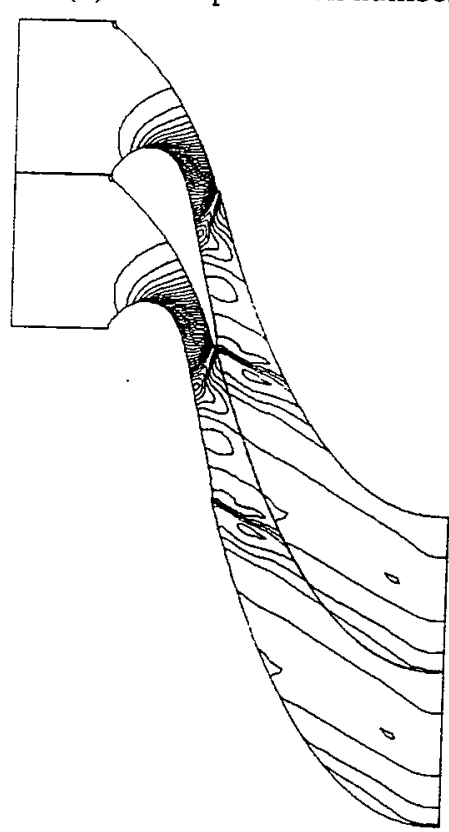

(f) Pressure contours of design

Figure 5. Design optimization of the VKI-LS82 turbine blade using ten design variables. 


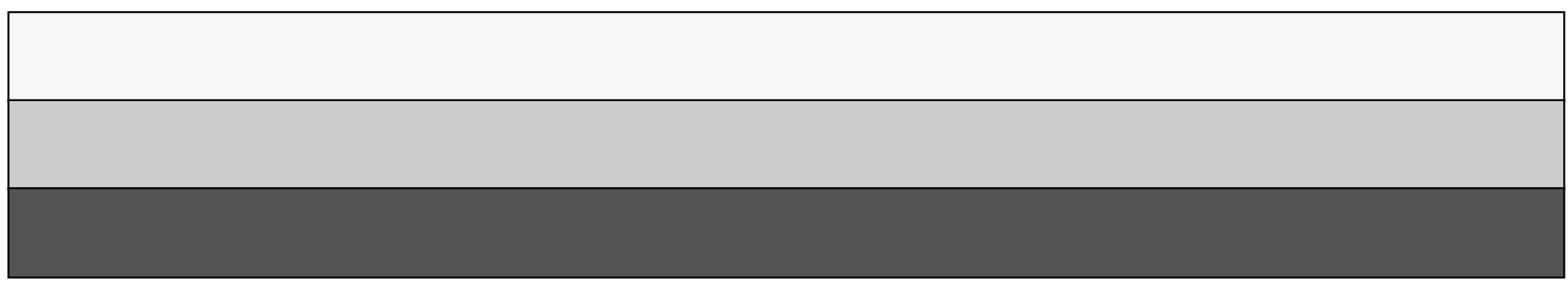

